\title{
Diabetes insipidus and panhypopituitarism in a neonate with congenital toxoplasmosis
}

Mirjana Danilović, Tatjana Milenković, Rade Vuković, Slađana Todorović, Jelena Martić, Katarina Pejić

Department of Endocrinology, Department of Neonatology, Mother and Child Health Care Institute, Belgrade, Serbia

\author{
Correspondence: \\ danilovic.mirjana@yahoo.com \\ Tel.: +381113108193 \\ Fax.: +381112060866
}

Received: December13, 2018

Accepted: January16, 2018

Key words: Congenital toxoplasmosis Diabetes insipidus - Neonate.
Objectives - Although congenital toxoplasmosis (CTox) is asymptomatic in most cases, some infants/neonates present with variable clinical manifestations, including anaemia, jaundice, hepatosplenomegaly, seizures, hydrocephalus, chorioretinitis and sensorineural deafness. Involvement of the hypothalamic-pituitary axis is rarely reported with CTox. Case report - We present a full term baby girl who was admitted to the hospital on the $15^{\text {th }}$ day of her life, due to an intense tremor and irritability. Brain ultrasound and CT exam revealed calcifications of the brain parenchyma and severe ventriculomegaly. Serological and molecular findings (positive specific immunoglobulin $\mathrm{M}$ and immunoglobulin $\mathrm{G}$ antibodies, polymerase chain reaction) confirmed CTox. After diagnosis was established, specific therapy with pyrimethamine, sulfodiazine and folic acid was started. On the fifth day of hospitalisation, laboratory findings revealed high serum sodium and chloride, high serum osmolality, low urine osmolality and low urine specific gravity. Since these findings strongly suggested the diagnosis of diabetes insipidus (DI) in this new born, further endocrinological workup was performed to assess the pituitary function. An ACTH (synacthen) stimulation test showed a low basal cortisol level with a positive response after administration of synacthen. The free thyroxin level was also low and repeated prolactin levels were high. On the basis of these findings, it was concluded that this new born has panhypopituitarism and diabetes insipidus. Hormonal substitution therapy with hydrocortisone, L-thyroxine and desmopressin was started and gradual normalization of electrolytes and hormonal status was noted. Conclusion - A continuous endocrinological monitoring of a child with CTox is important and necessary for early detection, timely therapy and prevention of severe sequelae.

\section{Introduction}

Congenital toxoplasmosis (CTox) results from the transplacental passage of the Toxoplasma gondii parasite from mother to the foetus. The risk of vertical transmission and the severity of foetal damage depends on the stage of pregnancy when the maternal infec- tion occurs. Although CTox is asymptomatic in most cases, some infants/neonates present with variable clinical manifestations, including anaemia, jaundice, hepatosplenomegaly, seizures, hydrocephalus, chorioretinitis and sensorineural hearing loss (1). Involvement of the hypothalamic-pituitary axis is rarely 
reported with CTox (2-6). It usually affects the anterior pituitary, leading to hypopituitarism. Exceptionally, the function of the posterior pituitary may be affected, with resulting diabetes insipidus.

In this case report a neonate with panhypopituitarism and diabetes insipidus, due to CTox is presented.

\section{Case report}

A full-term baby girl was born after an uneventful pregnancy. The new born was delivered vaginally with a birth weight of 3150 $\mathrm{g}$ and Apgar score of 9/9. The mother felt healthy during the pregnancy, with no signs of any infection or disease. Postnatal adaptation was normal, and the child was discharged as a healthy baby from the maternity hospital on the third day of life. On the $15^{\text {th }}$ postnatal day the neonate was admitted to the hospital ward due to an intense tremor and irritability. Parents noticed that the new born was irritable and had had trouble feeding during the few days before admission. For the previous several days, they had also noticed a tremor. On admission, the baby was tachypnoic, tachycardic, irritable and a severe tremor was also noted. The rest of the physical exam was normal. The standard laboratory workup for neonatal infection was normal. Brain ultrasound and CT scan were performed. The findings revealed calcifications of the brain parenchyma, mostly pronounced in the periventricular regions, with cystic changes of the white matter and severe ventriculomegaly (Fig.1).

Since these findings were highly indicative of a congenital infection with Toxoplasma gondii, additional serological and molecular testing of the mother and the neonate were conducted. Serology for Toxoplasma specific immunoglobulin $\mathrm{M}(\mathrm{IgM})$ performed in this neonate was $3.54 \mathrm{U} / \mathrm{ml}$ (positive $>0.65 \mathrm{U} /$ $\mathrm{ml}$ ), and immunoglobulin $\mathrm{G}$ (IgG) was 270 $\mathrm{U} / \mathrm{ml}$ (positive $>8 \mathrm{U} / \mathrm{ml}$ ). A high concentration was found of specific IgG antibodies that corresponded to those in the mother's blood (IgG $478 \mathrm{U} / \mathrm{ml}$, IgM $2.56 \mathrm{U} / \mathrm{ml}$ ). The polymerase chain reaction method was then performed, and the results confirmed the presence of parasite DNA in the blood of the new born. In view of these results, it was concluded that the new born had confirmed CTox and that the mother had probably been infected during the second trimes
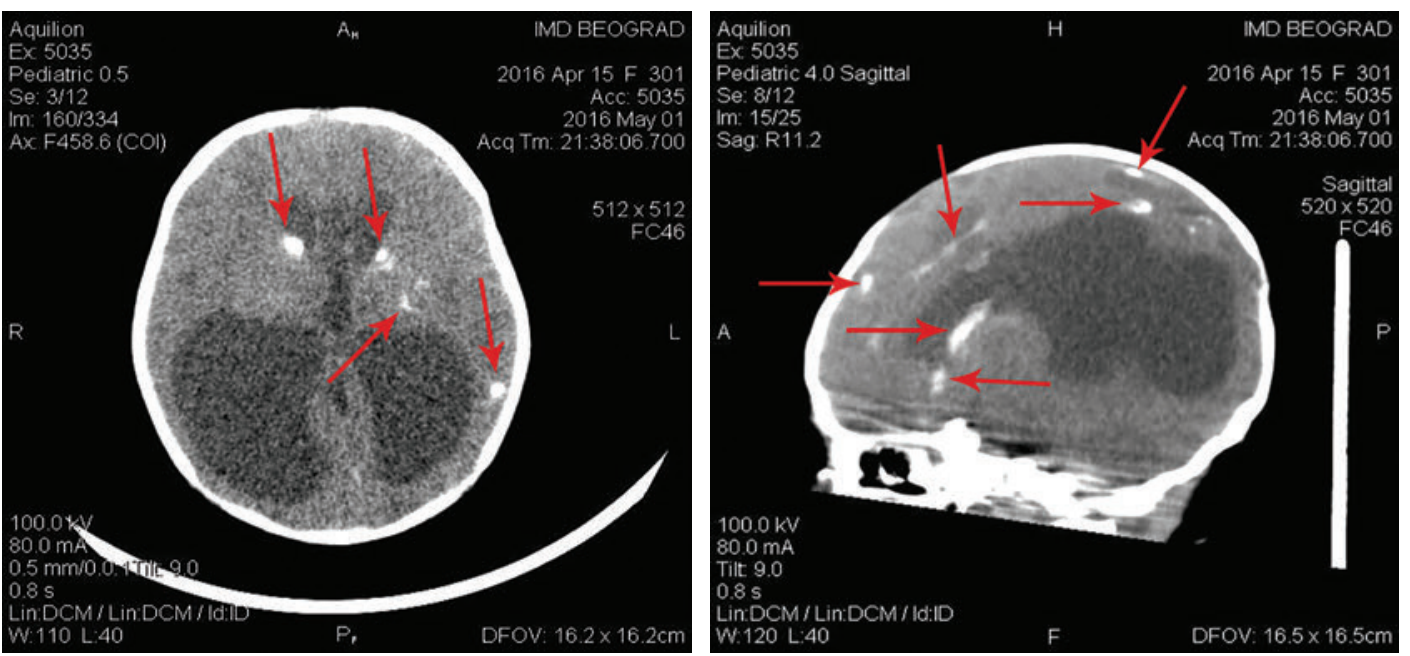

Fig. 1. Computerized tomography (a-coronal, b-sagittal images) showing extreme ventriculomegaly and calcification of the brain parenchyma. 
ter. After diagnosis was established, specific therapy with pyrimethamine, sulfodiazine and folic acid was started and, due to the high proteinorachia level $(>11 \mathrm{~g} / \mathrm{l})$, prednisone therapy was administered to control the inflammatory reaction.

On the fifth day of hospitalisation, significant weight loss with the accompanying clinical signs of dehydration were noted. Laboratory findings showed high serum sodium $(154 \mathrm{mmol} / \mathrm{l})$ and chloride (115 $\mathrm{mmol} / \mathrm{l}$ ) with potassium in the normal range. Serum osmolality was high $(318 \mathrm{mOsm} / \mathrm{kg})$ with inappropriately low urine osmolality $(259 \mathrm{mOsm} / \mathrm{kg})$, and urine specific gravity of $<1005$. Since these findings strongly suggested the diagnosis of diabetes insipidus (DI) in this new born, with confirmed CNS damage, further endocrinological workup was performed to assess the pituitary function. The ACTH (synacthen) stimulation test showed a low basal cortisol level $(15 \mathrm{nmol} / \mathrm{l})$, with a positive response up to $522 \mathrm{nmol} / \mathrm{l}$ after administration of synacthen. The free thyroxin level of $8 \mathrm{pmol} / \mathrm{l}$ was also low (normal range $10.6-39.8 \mathrm{pmol} / \mathrm{l}$ ), with an inappropriately normal level of thyro-stimulating hormone (3.54 mIU/l). Repeated prolactin levels were high, up to $4139 \mathrm{mIU} / \mathrm{l}$. On the basis of these findings, it was concluded that this new born, alongside extensive brain lesions due to CTox, had panhypopituitarism and diabetes insipidus. The most likely mechanism in this case was the hypothalamic lesion or the complete interruption of the hypothalamic-pituitary axis, due to the inflammatory reaction to parasites in the CNS. After the diagnoses of panhypopituitarism and DI had been established, hormonal substitution therapy with hydrocortisone, L-thyroxine and desmopressin was started. In the further course, gradual normalisation of electrolytes and hormonal status was noted.

\section{Discussion}

Congenital toxoplasmosis may in rare cases affect the hypothalamic pituitary axis, resulting in deficiency of the anterior and, more rarely, the posterior pituitary hormones (28). Our patient presented with panhypopituitarism and diabetes insipidus. Although there are several reports of rare cases of anterior pituitary disturbances in CTox, diabetes insipidus is an exceptionally rare complication of CTox. Massa et al. (3) reported three cases of CTox and hypothalamic-pituitary dysfunction, all of which presented with different patterns of combined hormonal deficiencies (GH deficiency, diabetes insipidus, tertiary hypothyroidism, ACTH insufficiency, and delayed puberty). Karadag et al. (7) reported a neonate with CTox and hydrocephalus, who developed DI on the $10^{\text {th }}$ postnatal day. Similarly, Oygur et al. (8) presented a 33-day-old boy who had polyuria, hypernatremia, and dehydration secondary to central DI, associated with CTox. Siahanidou et al. (9) described a new born who presented with prolonged fever and was confirmed later to have central diabetes insipidus, hypothyroidism, and ACTH deficiency. Our patient had disturbance of the entire hypothalamic-pituitary axis: central diabetes insipidus, central hypothyroidism, ACTH insufficiency and hyperprolactinaemia. Including the case of CTox presented in this article, all the described cases highlight the fact that hypothalamic-pituitary dysfunction does occur in CTox, and that the disorders might not be apparent at the time of establishing the CTox diagnosis and could even develop over various amounts of time. Thus, infants and children with confirmed CTox should be monitored for disorders of hypothalamic-pituitary function, even if the initial findings are normal. 


\section{Conclusion}

The characteristic clinical picture of CTox in a 15-day-old new born is presented in this case report. Brain ultrasound and CT findings suggested the diagnosis, which was confirmed using serological and molecular tests. Diagnosis was made and treatment started within the first few days of hospitalisation. Studies have shown that endocrine complications are rare but have serious potential implications (2-6). These complications may present in neonatal period, but also at a later age, during growth and development. Therefore, continuous endocrinological monitoring of a child with CTox is important and necessary for early detection, timely therapy and prevention of severe sequelae. This case report also highlights the importance of antenatal and neonatal screening for toxoplasmosis. It offers the possibility of early diagnosis and treatment of the affected mother and foetus, thus reducing the risk of vertical transmission, and, in cases of transmission of the infection to the foetus, minimizes the complications $(1,10-12)$.

Conflict of interest: The authors declare that they have no conflict of interest.

Authors' contributions: Conception and design: MD and RV; Acquisition, analysis and interpretation of data: MD, RV, and TM; Drafting the article: MD; Revising the article critically for intellectual content: $\mathrm{MD}$, TM, RV, ST, JM, KP; Approved final version of the manuscript: MD, TM, RV, ST, JM, KP.

\section{References}

1. SYROCOT (Systematic Review on Congenital Toxoplasmosis) study group. Effectiveness of prenatal treatment for congenital toxoplasmosis. Lancet. 2007;369(9556):115.
2. Suresh Babu PS, Nagendra K, Navaz RS, Ravindranath HM. Congenital toxoplasmosis presenting as hypogonadotropic hypogonadism. Indian J Pediatr. 2007;74:577-9.

3. Massa G, Vanderschueren-Lodeweyckx M, Van Vliet G, Craen M, de Zegher F, Eggermont E. Hypothalamo-pituitary dysfunction in congenital toxoplasmosis. Eur J Pediatr. 1989;148(8):742-4.

4. Robinson A, Verbalis J. Posterior pituitary. In: Kronenberg H. editor. Williams Textbook of Endocrinology, 11th ed. Philadephia: Saunders Elsevier; 2008. p. 263-287.

5. Ghirardello S, Garre ML, Rossi A, Maghnie M. The diagnosis of childrenwith central diabetes insipidus. J Pediatr Endocrinol Metab. 2007;20:35975.

6. Di Iorgi N, Napoli F, Allegri A, Olivieri I, Bertelli E, Gallizia A, et al. Diabetes insipidus - diagnosis and management. Horm Res Paediatr. 2012;77:6984.

7. Karadag A, Erdeve O. Isolated central diabetes insipidus in a newborn with congenital toxoplasmosis. J Pediar Endocrinol Metab. 2006;19(2):173-5.

8. Oygür N, Yilmaz G, Ozkaynak C, Guven AG. Central diabetes insipidus in a patient with congenital toxoplasmosis. Am J of Perin. 1998;15(3):191-2.

9. Siahanidou T, Tsoumas D, Kanaka-Gantenbein C, Mandyla H. Neuroendocrine abnormalities in a neonate with congenital toxoplasmosis. J Pediatr Endocrinol Metab. 2006;19(11):1363-6.

10. Robert-Gangneux F, Dardé ML. Epidemiology of and diagnostic strategies for toxoplasmosis. Clin Microbiol Rev. 2012;25(2):264.

11. McLeod R, Boyer K, Karrison T, Kasza T, Swisher C, Roizen N, et al. Outcome of treatment for congenital toxoplasmosis 1981-2004: the National Collaborative Chicago-Based. Congenital Toxoplasmosis Study. Clin Infect Dis. 2006;42:1383.

12. Kieffer F, Wallon M, Garcia P, Thulliez P, Peyron F, Frank J. Risk factors for retinochoroiditis during the first 2 years of life in infants with treated congenital toxoplasmosis. Pediatr Infect Dis J. 2008;2008(27):27-32. 